Bull. Korean Math. Soc. 39 (2002), No. 3, pp. 359-376

\title{
DARBOUX TRANSFORMS AND ORTHOGONAL POLYNOMIALS
}

\author{
GANG JOON YOON
}

\begin{abstract}
We give a new interpretation of Darboux transforms in the context of orthogonal polynomials and find conditions in order for any Darboux transform to yield a new set of orthogonal polynomials. We also discuss connections between Darboux transforms and factorization of linear differential operators which have orthogonal polynomial eigenfunctions.
\end{abstract}

\section{Introduction}

Let $\mathbb{P}$ be the space of all polynomials in one variable with complex coefficients and denote the degree of a polynomial $\pi(x)$ by $\operatorname{deg}(\pi)$ with the convention that $\operatorname{deg}(0)=-1$. By a polynomial system(PS), we mean a sequence of polynomials $\left\{\phi_{n}(x)\right\}_{n=0}^{\infty}$ with $\operatorname{deg}\left(\phi_{n}\right)=n, n \geq 0$.

We call any linear functional $\sigma$ on $\mathbb{P}$ a moment functional and denote its action on a polynomial $\pi(x)$ by $\langle\sigma, \pi\rangle$. For a moment functional $\sigma$, we call

$$
\sigma_{n}:=\left\langle\sigma, x^{n}\right\rangle, \quad n=0,1, \cdots
$$

the $n$-th moment of $\sigma$. We say that a moment functional $\sigma$ is quasidefinite if its moments $\left\{\sigma_{n}\right\}_{n=0}^{\infty}$ satisfy the Hamburger condition

$$
\Delta_{n}(\sigma):=\operatorname{det}\left[\sigma_{i+j}\right]_{i, j=0}^{n} \neq 0, \quad n \geq 0 .
$$

Any PS $\left\{\phi_{n}(x)\right\}_{n=0}^{\infty}$ determines a moment functional $\sigma$ (uniquely up to a non-zero constant multiple), called a canonical moment functional of $\left\{\phi_{n}(x)\right\}_{n=0}^{\infty}$, by the conditions

$$
\left\langle\sigma, \phi_{0}\right\rangle \neq 0 \quad \text { and } \quad\left\langle\sigma, \phi_{n}\right\rangle=0, \quad n \geq 1 .
$$

Received January 8, 2002

2000 Mathematics Subject Classification: 33C45, 34A99.

Key words and phrases: Darboux transform, orthogonal polynomials, differential equations.

This work was supported in part by BK21 project. 
A PS $\left\{P_{n}(x)\right\}_{n=0}^{\infty}$ is called an orthogonal polynomial system (OPS) if there is a moment functional $\sigma$ such that

$$
\left\langle\sigma, P_{m} P_{n}\right\rangle=K_{n} \delta_{m n}, \quad m, n \geq 0,
$$

where $K_{n}$ are non-zero constants and $\delta_{m n}$ is the Kronecker delta function. In this case, we say that $\left\{P_{n}(x)\right\}_{n=0}^{\infty}$ is an OPS relative to $\sigma$ and $\sigma$ must be a canonical moment functional of $\left\{P_{n}(x)\right\}_{n=0}^{\infty}$.

For a moment functional $\sigma$, a polynomial $\pi(x)$, and a complex number $\lambda$, we let $\sigma^{\prime}, \pi \sigma$, and $(x-\lambda)^{-1} \sigma$ be the moment functionals defined by

$$
\left\langle\sigma^{\prime}, \phi\right\rangle=-\left\langle\sigma, \phi^{\prime}\right\rangle, \quad\langle\pi \sigma, \phi\rangle=\langle\sigma, \pi \phi\rangle,
$$

and

$$
\left\langle(x-\lambda)^{-1} \sigma, \phi(x)\right\rangle=\left\langle\sigma, \frac{\phi(x)-\phi(\lambda)}{x-\lambda}\right\rangle
$$

for every polynomial $\phi(x)$.

The following results are immediate consequences of these definitions.

LEMMA 1.1. ([10] and [11]) Let $\sigma$ and $\tau$ be moment functionals and $\lambda$ a complex number. Then

(i) $(x-\lambda) \sigma=\tau$ if and only if

$$
\sigma=(x-\lambda)^{-1} \tau+\sigma_{0} \delta(x-\lambda) .
$$

(ii) If $\sigma$ is quasi-definite and $\left\{P_{n}(x)\right\}_{n=0}^{\infty}$ is an OPS relative to $\sigma$, then $\left\langle\tau, P_{n}\right\rangle=0, \quad n \geq k+1$ for some integer $k \geq 0$ if and only if $\tau=\phi(x) \sigma$ for some polynomial $\phi(x)$ of degree $\leq k$.

Due to Favard's theorem [2], a monic PS $\left\{P_{n}(x)\right\}_{n=0}^{\infty}$ is a monic OPS (MOPS in short) if and only if $\left\{P_{n}(x)\right\}_{n=0}^{\infty}$ satisfies a three-term recurrence relation:

$$
P_{n+1}(x)=\left(x-b_{n}\right) P_{n}(x)-c_{n} P_{n-1}(x), \quad n \geq 0, \quad\left(P_{-1}(x)=0\right),
$$

where $\left\{b_{n}\right\}_{n=0}^{\infty}$ and $\left\{c_{n}\right\}_{n=0}^{\infty}$ are complex numbers with $c_{n} \neq 0, n \geq 1$.

We then let $\left\{P_{n}^{(1)}(x)\right\}_{n=0}^{\infty}$ be the monic numerator OPS satisfying

$$
\begin{aligned}
& P_{n+1}^{(1)}(x)=\left(x-b_{n+1}\right) P_{n}^{(1)}(x)-c_{n+1} P_{n-1}^{(1)}(x), \quad n \geq 0, \\
& \left(P_{-1}^{(1)}(x)=0, P_{0}^{(1)}(x)=1\right)
\end{aligned}
$$

and $\left\{P_{n}(c ; x)\right\}_{n=0}^{\infty}$ the monic co-recursive OPS with parameter $c \in \mathbb{C}$ ([3]) satisfying

$$
P_{n}(c ; x)=P_{n}(x)-c P_{n-1}^{(1)}(x), \quad n \geq 0 .
$$

In a series of papers $([4,5,6,7])$, Grünbaum and et al introduced Darboux transform to analyze and extend classical results by Bochner 
[1], Krall [8], and Littlejohn [14] on the bispectral problem of classifying all Bochner-Krall orthogonal polynomials, that is, PS's which satisfy the second order difference equation (1.2) and are eigenfunctions of some linear differential operator.

Darboux transform consists of factoring the second order difference operator (i.e., the Jacobi matrix) induced by the three-term recurrence relation (1.2) into a product of two first order difference operators. One then changes the order of these two factors, by which one can obtain a new second order difference operator which depends on one or two free parameters.

In particular, Grünbaum and Heine [4] showed how one can obtain the so-called "Krall polynomials" by one or two Darboux transforms applied to classical orthogonal polynomials. In [4], Grünbaum and Heine showed this fact by working out each one of the Krall polynomials case by case.

In fact, the newly obtained second order difference operator by Darboux transform may or may not yield a new set of orthogonal polynomials, depending on the choice of free parameters involved. In this work, we first find conditions under which a Darboux transform yields a new OPS. We also interpret Darboux transform via moment functionals, which shows very clearly the role of Darboux transform in the context of orthogonal polynomials.

This new interpretation of Darboux transform via moment functionals can explain the result [4] in a unified way as well as others.

Lastly, we also discuss some connections between Darboux transforms and factorization of linear differential operators which have orthogonal polynomial eigenfunctions.'

\section{Darboux transform}

From now on, we always let $\left\{P_{n}(x)\right\}_{n=0}^{\infty}$ be an MOPS relative to a quasi-definite moment functional $\sigma$, which satisfies the three-term recurrence relation

$$
\begin{aligned}
& P_{n+1}(x)=\left(x-b_{n}\right) P_{n}(x)-c_{n} P_{n-1}(x), \quad n \geq 0 \\
& \left(P_{-1}(x)=0, P_{0}(x)=1\right)
\end{aligned}
$$

where $c_{n} \neq 0, n \geq 1$. We can express (2.2) as

$$
J P=x P,
$$


where $P:=\left(P_{0}(x), P_{1}(x), P_{2}(x), \cdots\right)^{t}$ and the tridiagonal semi-infinite matrix

$$
J=\left(\begin{array}{ccccc}
b_{0} & 1 & 0 & 0 & \ldots \\
c_{1} & b_{1} & 1 & 0 & \ldots \\
0 & c_{2} & b_{2} & 1 & \ldots \\
\vdots & \vdots & \vdots & \vdots & \ddots
\end{array}\right)
$$

is the Jacobi matrix of the MOPS $\left\{P_{n}(x)\right\}_{n=0}^{\infty}$. Now for any $\lambda \in \mathbb{C}$, decompose $J$ into

$$
J=A B+\lambda I \quad \text { or } \quad J=B A+\lambda I
$$

whenever it is possible, where

$$
A=\left(\begin{array}{ccccc}
\alpha_{0} & 1 & 0 & 0 & \ldots \\
0 & \alpha_{1} & 1 & 0 & \ldots \\
0 & 0 & \alpha_{2} & 1 & \ldots \\
\vdots & \vdots & \vdots & \vdots & \ddots
\end{array}\right) \text { and } B=\left(\begin{array}{ccccc}
1 & 0 & 0 & 0 & \ldots \\
\beta_{1} & 1 & 0 & 0 & \ldots \\
0 & \beta_{2} & 1 & 0 & \ldots \\
\vdots & \vdots & \vdots & \vdots & \ddots
\end{array}\right)
$$

are lower and upper triangular matrices.

In either case, we call

$$
\tilde{J}=B A+\lambda I \quad(\text { or } \tilde{J}=A B+\lambda I)
$$

a Darboux transform of $J$ with parameter $\lambda$.

In the followings, for simplicity of notation, we use $\left[\alpha_{n}, \beta_{n}, \gamma_{n}\right]$ to denote the tridiagonal semi-infinite matrix

$$
\left[\alpha_{n}, \beta_{n}, \gamma_{n}\right]=\left(\begin{array}{ccccc}
\beta_{0} & \gamma_{0} & 0 & 0 & \ldots \\
\alpha_{1} & \beta_{1} & \gamma_{1} & 0 & \ldots \\
0 & \alpha_{2} & \beta_{2} & \gamma_{2} & \ldots \\
\vdots & \vdots & \vdots & \vdots & \ddots
\end{array}\right)
$$

It is then easy to see that $\tilde{J}=\left[\tilde{c}_{n}, \tilde{b}_{n}, 1\right]$ is also a tridiagonal semi-infinite matrix, which naturally induces another monic PS $\left\{\tilde{P}_{n}(x)\right\}_{n=0}^{\infty}$ defined recursively by the three-term recurrence relation

$$
\begin{aligned}
& \tilde{P}_{n+1}(x)=\left(x-\tilde{b}_{n}\right) \tilde{P}_{n}(x)-\tilde{c}_{n} \tilde{P}_{n-1}(x), \quad n \geq 0, \\
& \left(\tilde{P}_{-1}(x)=0, \tilde{P}_{0}(x)=1\right) .
\end{aligned}
$$

We call $\left\{\tilde{P}_{n}(x)\right\}_{n=0}^{\infty}$ a Darboux transform of $\left\{P_{n}(x)\right\}_{n=0}^{\infty}$ with parameter $\lambda$. By Farvard theorem ([2]), $\left\{\tilde{P}_{n}(x)\right\}_{n=0}^{\infty}$ is an MOPS if and only if $\tilde{c}_{n} \neq 0$ for $n \geq 1$.

We now have a natural question: When can we decompose the Jacobi matrix as in (2.3) so that $\left\{\tilde{P}_{n}(x)\right\}_{n=0}^{\infty}$ is also an MOPS? 
Case 1: Decomposition $J=A B+\lambda I$.

Assume that $J$ can be decomposed into

$$
J=A B+\lambda I .
$$

Then (2.6) means $\left[c_{n}, b_{n}, 1\right]=\left[\alpha_{n} \beta_{n}, \alpha_{n}+\beta_{n+1}+\lambda, 1\right]$, that is

$$
\beta_{n}=b_{n-1}-\alpha_{n-1}-\lambda, n \geq 1 \text { and } \alpha_{n}=c_{n} / \beta_{n}, \quad n \geq 1 .
$$

The relation (2.7) implies that we have two free parameters $\alpha_{0}$ and $\lambda$ from which all $\left\{\alpha_{n}\right\}_{n=1}^{\infty}$ and $\left\{\beta_{n}\right\}_{n=1}^{\infty}$ are uniquely determined as long as $\beta_{n} \neq 0, n \geq 1$ (so that $\alpha_{n} \neq 0, n \geq 1$ ). In this case $\tilde{J}=B A+\lambda I=$ $\left[\tilde{c}_{n}, \tilde{b}_{n}, 1\right]$, where

$$
\tilde{c}_{n}=\alpha_{n-1} \beta_{n}, n \geq 1 \text { and } \tilde{b}_{n}=\alpha_{n}+\beta_{n}+\lambda, n \geq 0\left(\beta_{0}=0\right) \text {. }
$$

Hence, $\left\{\tilde{P}_{n}(x)\right\}_{n=0}^{\infty}$ is an MOPS if and only if $\tilde{c}_{n}=\alpha_{n-1} \beta_{n} \neq 0, n \geq 1$.

LEMma 2.1. If $J=A B+\lambda I$, then

$$
\tilde{P}_{n}(x)=P_{n}(x)+\beta_{n} P_{n-1}(x), \quad n \geq 0
$$

and

$$
(x-\lambda) P_{n}(x)=\tilde{P}_{n+1}(x)+\alpha_{n} \tilde{P}_{n}(x), \quad n \geq 0 .
$$

Proof. Define a monic PS $\left\{Q_{n}(x)\right\}_{n=0}^{\infty}$ by

$$
Q=B P, \quad \text { that is, } \quad Q_{n}(x)=P_{n}(x)+\beta_{n} P_{n-1}(x), \quad n \geq 0 .
$$

Then we have by (2.6)

$$
(x-\lambda) P=(J-\lambda I) P=A B P=A Q,
$$

that is,

$$
(x-\lambda) P_{n}(x)=Q_{n+1}+\alpha_{n} Q_{n}(x), \quad n \geq 0 .
$$

We also have

$$
B A Q=(x-\lambda) B P=(x-\lambda) Q,
$$

which means that $\left\{Q_{n}(x)\right\}_{n=0}^{\infty}$ also satisfy the three-term recurrence relation (2.6) with the initial conditions $Q_{-1}(x)=0$ and $Q_{0}(x)=1$. Hence $Q_{n}(x)=\tilde{P}_{n}(x), n \geq 0$, which completes the proof.

LEMMA 2.2. Let $\left\{P_{n}(x)\right\}_{n=0}^{\infty}$ be an MOPS relative to $\sigma$ satisfying (2.2). Then $\tilde{\sigma}=(x-\lambda) \sigma(\lambda \in \mathbb{C})$ is also quasi-definite if and only if $P_{n}(\lambda) \neq 0, n \geq 1$.

When $\tilde{\sigma}$ is also quasi-definite, its corresponding MOPS $\left\{\tilde{P}_{n}(x)\right\}_{n=0}^{\infty}$ satisfy

$$
(x-\lambda) \tilde{P}_{n}(x)=P_{n+1}(x)-\frac{P_{n+1}(\lambda)}{P_{n}(\lambda)} P_{n}(x), \quad n \geq 0
$$




$$
P_{n}(x)=\tilde{P}_{n}(x)-\frac{P_{n-1}(\lambda)}{P_{n}(\lambda)} c_{n} \tilde{P}_{n-1}(x), \quad n \geq 0 .
$$

Proof. Assume $\tilde{\sigma}=(x-\lambda) \sigma$ is quasi-definite but $P_{n}(\lambda)=0$ for some $n \geq 1$. Then $P_{n}(x)=(x-\lambda) \pi_{n-1}(x)$, where $\pi_{n-1}(x)$ is a monic polynomial of degree $n-1$. Hence

$$
\left\langle\sigma, P_{n}^{2}(x)\right\rangle=\left\langle\sigma, P_{n}(x) \tilde{P}_{n}(x)\right\rangle=\left\langle\tilde{\sigma}, \pi_{n-1}(x) \tilde{P}_{n}(x)\right\rangle=0,
$$

which is impossible. Hence, $P_{n}(\lambda) \neq 0, n \geq 1$.

Conversely assume $P_{n}(\lambda) \neq 0, n \geq 1$ and define a monic PS $\left\{\tilde{P}_{n}(x)\right\}_{n=0}^{\infty}$ by $(2.11)$. Then

$$
\left\langle\tilde{\sigma},(x-\lambda)^{k} \tilde{P}_{n}(x)\right\rangle=-\frac{P_{n+1}(\lambda)}{P_{n}(\lambda)}\left\langle\sigma,(x-\lambda)^{k} P_{n}(x)\right\rangle, \quad 0 \leq k \leq n
$$

so that $\left\{\tilde{P}_{n}(x)\right\}_{n=0}^{\infty}$ is an MOPS relative to $\tilde{\sigma}=(x-\lambda) \sigma$. Finally $(2.12)$ comes from (2.11) by Christoffel-Darboux identity.

The MOPS $\left\{\tilde{P}_{n}(x)\right\}_{n=0}^{\infty}$ in Lemma 2.2 is called the monic kernel polynomials of $\left\{P_{n}(x)\right\}_{n=0}^{\infty}$ with $K$-parameter $\lambda([2])$.

Proposition 2.3. Let $\left\{P_{n}(x)\right\}_{n=0}^{\infty}$ be the MOPS relative to $\sigma$ satisfying (2.2). Define a new monic PS $\left\{\tilde{P}_{n}(x)\right\}_{n=0}^{\infty}$ by $(2.9)$, where $\left\{\beta_{n}\right\}_{n=1}^{\infty}$ are arbitrary constants with $\beta_{1} \neq 0$. Then the followings are all equivalent.

(i) $\left\{\tilde{P}_{n}(x)\right\}_{n=0}^{\infty}$ is also an MOPS;

(ii) $\beta_{n} \neq 0$ for $n \geq 1, b_{n}-\beta_{n+1}-\frac{c_{n}}{\beta_{n}}:=\lambda$ (constant) for $n \geq 1$, and $c:=\lambda+\beta_{1}-b_{0} \neq 0$

(iii) there are constants $\lambda$ and $c \neq 0$ such that

$$
P_{n}(c ; \lambda)=P_{n}(\lambda)-c P_{n-1}^{(1)}(\lambda) \neq 0, \quad \dot{n} \geq 0
$$

and

$$
\beta_{n}=-\frac{P_{n}(c ; \lambda)}{P_{n-1}(c ; \lambda)}, \quad n \geq 1 ;
$$

(iv) there is a constant $\lambda$ such that $(x-\lambda) \tilde{\sigma}=\sigma$ and

$$
\beta_{n}=-\frac{\left\langle\tilde{\sigma}, P_{n}\right\rangle}{\left\langle\tilde{\sigma}, P_{n-1}\right\rangle}, \quad n \geq 1,
$$

where $\tilde{\sigma}$ is a canonical moment functional of $\left\{\tilde{P}_{n}(x)\right\}_{n=0}^{\infty}$. 
Moreover, in this case, we have $\tilde{P}_{n}(\lambda) \neq 0, n \geq 1$ and

$$
(x-\lambda) P_{n}(x)=\tilde{P}_{n+1}(x)-\frac{\tilde{P}_{n+1}(\lambda)}{\tilde{P}_{n}(\lambda)} \tilde{P}_{n}(x), \quad n \geq 0 .
$$

Proof. For the equivalence of (i), (i), and (iii), see Theorem 2 in [15] (see also Theorem 4.2 in [10]). Assume now that $\left\{\tilde{P}_{n}(x)\right\}_{n=0}^{\infty}$ is an MOPS relative to $\tilde{\sigma}$. Then

$$
\left\langle\tilde{\sigma}, \tilde{P}_{n}(x)\right\rangle=\left\langle\tilde{\sigma}, P_{n}(x)\right\rangle+\beta_{n}\left\langle\tilde{\sigma}, P_{n-1}(x)\right\rangle=0, \quad n \geq 1
$$

so that $\left\langle\tilde{\sigma}, P_{n}(x)\right\rangle \neq 0, n \geq 0$ and $\beta_{n}=-\frac{\left\langle\tilde{\sigma}, P_{n}(x)\right\rangle}{\left\langle\tilde{\sigma}, P_{n-1}(x)\right\rangle}, n \geq 1$. On the other hand,

$$
\left\langle\sigma, \tilde{P}_{n}(x)\right\rangle=\left\langle\sigma, P_{n}(x)\right\rangle+\beta_{n}\left\langle\sigma, P_{n-1}(x)\right\rangle=0, \quad n \geq 2
$$

so that $\sigma=(a x+b) \tilde{\sigma}$ by Lemma 1.1 (ii). From $\left\langle\sigma, \tilde{P}_{n}(x)\right\rangle=\langle\tilde{\sigma},(a x+$ b) $\left.\tilde{P}_{n}(x)\right\rangle$ for $n=0$, 1 , we obtain

$$
a=\frac{\beta_{1} \sigma_{0}}{\left\langle\tilde{\sigma}, x \tilde{P}_{1}(x)\right\rangle}=-\frac{1}{c} \frac{\sigma_{0}}{\tilde{\sigma}_{0}} \quad \text { and } \quad b=\left(1+\frac{b_{0}-\beta_{1}}{c}\right) \frac{\sigma_{0}}{\tilde{\sigma}_{0}} .
$$

Hence, if we normalize $\tilde{\sigma}$ so that $\tilde{\sigma}_{0}:=\langle\tilde{\sigma}, 1\rangle=-\sigma_{0} / c$, then $a=1, b=$ $-\lambda$ so that $(x-\lambda) \tilde{\sigma}=\sigma$. Therefore, (i) implies (iv). Conversely, assume that (iv) holds. Then we can see easily that

$$
\begin{aligned}
& \left\langle\tilde{\sigma},(x-\lambda)^{k} \tilde{P}_{n}(x)\right\rangle \\
& =\left\{\begin{array}{cll}
0, & \text { if } \quad k=0 \text { and } n \geq 1 \\
-\frac{\left\langle\tilde{\sigma}, P_{n}(x)\right\rangle}{\left\langle\tilde{\sigma}, P_{n-1}(x)\right\rangle}\left\langle\sigma, P_{n-1}^{2}(x)\right\rangle \delta_{n k}, & \text { if } 1 \leq k \leq n .
\end{array}\right.
\end{aligned}
$$

Hence, $\left\{\tilde{P}_{n}(x)\right\}_{n=0}^{\infty}$ is an MOPS relative to $\tilde{\sigma}$. Therefore, (iv) implies (i).

Finally, assume that $\tilde{\sigma}$ is quasi-definite. Then $\tilde{P}_{n}(\lambda) \neq 0, n \geq 1$ and (2.13) holds by Lemma 2.2 (where the roles of $\sigma$ and $\tilde{\sigma}$ are exchanged).

Theorem 2.4. The Jacobi matrix $J=\left[c_{n}, b_{n}, 1\right]$ of the MOPS $\left\{P_{n}(x)\right\}_{n=0}^{\infty}$ can be decomposed into $J=A B+\lambda I$ as in (2.3) so that the corresponding Darboux transform $\left\{\tilde{P}_{n}(x)\right\}_{n=0}^{\infty}$ is also an MOPS if and only if the two free parameters $\alpha_{0}$ and $\lambda$ are chosen so that

$$
\alpha_{0} \neq 0 \quad \text { and } \quad P_{n}\left(-\alpha_{0} ; \lambda\right)=P_{n}(\lambda)+\alpha_{0} P_{n-1}^{(1)}(\lambda) \neq 0, \quad n \geq 0
$$


Moreover, in this case, $\left\{\tilde{P}_{n}(x)\right\}_{n=0}^{\infty}$ is orthogonal relative to $\tilde{\sigma}$ satisfying $(x-\lambda) \tilde{\sigma}=\sigma$ and

$$
\beta_{n}=-\frac{P_{n}\left(-\alpha_{0} ; \lambda\right)}{P_{n-1}\left(-\alpha_{0} ; \lambda\right)}, \quad n \geq 1 \quad \text { and } \quad \alpha_{n}=-\frac{\tilde{P}_{n+1}(\lambda)}{\tilde{P}_{n}(\lambda)}, \quad n \geq 0 .
$$

Proof. Assume that we can decompose $J$ as $J=A B+\lambda I$ so that $\tilde{J}=B A+\lambda I$ induces an MOPS $\left\{\tilde{P}_{n}(x)\right\}_{n=0}^{\infty}$. Then we have (2.9) and (2.10). Hence by (2.13)

$$
\alpha_{n}=-\frac{\tilde{P}_{n+1}(\lambda)}{\tilde{P}_{n}(\lambda)}, \quad n \geq 0 .
$$

In particular, $\alpha_{0}=-\tilde{P}_{1}(\lambda)=-\left(\lambda-b_{0}+\beta_{1}\right)=-c$ so that $(2.14)$ and (2.15) come from Proposition 2.3. Conversely, assume that (2.14) holds. Let

$$
\beta_{n}=-\frac{P_{n}\left(-\alpha_{0} ; \lambda\right)}{P_{n-1}\left(-\alpha_{0} ; \lambda\right)}, \quad n \geq 1 \quad \text { and } \quad \alpha_{n}=\frac{c_{n}}{\beta_{n}}, \quad n \geq 1 .
$$

Then by Proposition 2.3, $b_{n}-\beta_{n+1}-\alpha_{n}=\lambda, n \geq 1$ so that (2.7) holds. Hence $J=A B+\lambda I$ and $\left\{\tilde{P}_{n}(x)\right\}_{n=0}^{\infty}$ is also an MOPS.

Case 2 : Decomposition $J=B A+\lambda I$.

Assume that $J$ can be decomposed into

$$
J=B A+\lambda I
$$

which means

$$
\alpha_{n}=b_{n}-\beta_{n}-\lambda, \quad n \geq 0 \quad\left(\beta_{0}=0\right) \quad \text { and } \quad \beta_{n}=\frac{c_{n}}{\alpha_{n-1}}, \quad n \geq 1 .
$$

The relation (2.17) implies that we have only one free parameter $\lambda$ from which all $\left\{\alpha_{n}\right\}_{n=0}^{\infty}$ and $\left\{\beta_{n}\right\}_{n=1}^{\infty}$ are determined uniquely as long as $\alpha_{n} \neq$ 0 for $n \geq 0$ (so that $\beta_{n} \neq 0$ for $n \geq 1$ ). In this case the Darboux transform is $\tilde{J}=A B+\lambda I=\left[\tilde{c}_{n}, \tilde{b}_{n}, 1\right]$, where

$$
\tilde{c}_{n}=\alpha_{n} \beta_{n}, \quad n \geq 1 \quad \text { and } \quad \tilde{b}_{n}=\alpha_{n}+\beta_{n+1}+\lambda, n \geq 0 .
$$

Unlike as in Case 1, the Darboux transform $\left\{\tilde{P}_{n}(x)\right\}_{n=0}^{\infty}$ in Case 2 is an MOPS as long as the decomposition $J=B A+\lambda I$ is possible.

LEMMA 2.5. If $J=B A+\lambda I$, then

$$
P_{n}(x)=\tilde{P}_{n}(x)+\beta_{n} \tilde{P}_{n-1}(x), \quad n \geq 0
$$


and

$$
(x-\lambda) \tilde{P}_{n}(x)=P_{n+1}(x)+\alpha_{n} P_{n}(x), \quad n \geq 0 .
$$

Proof. Set $Q_{n+1}(x)=P_{n+1}(x)+\alpha_{n} P_{n}(x), n \geq 0$. Then

$$
(x-\lambda) P=(J-\lambda) P=B A P=B\left(Q_{1}(x), Q_{2}(x), Q_{2}(x), \cdots\right)^{t}
$$

or equivalently,

$$
(x-\lambda) P_{n}(x)=Q_{n+1}(x)+\beta_{n} Q_{n}(x), \quad n \geq 0 \quad\left(\beta_{0}=0\right) .
$$

Hence $Q_{n}(\lambda)=0, n \geq 1$ since $\beta_{n} \neq 0, n \geq 1$ so that

$$
Q_{n}(x)=(x-\lambda) \tilde{Q}_{n-1}(x), \quad n \geq 1,
$$

where $\left\{\tilde{Q}_{n}(x)\right\}_{n=0}^{\infty}$ is a monic PS. Then $(x-\lambda) \tilde{Q}=A P=A B Q$, that is,

$\tilde{Q}_{n+1}(x)=\left(x-\tilde{b}_{n}\right) \tilde{Q}_{n}(x)-\tilde{c}_{n} \tilde{Q}_{n-1}(x), n \geq 0\left(\tilde{Q}_{-1}(x)=0, \tilde{Q}_{0}(x)=1\right)$.

Hence $\tilde{Q}_{n}(x)=\tilde{P}_{n}(x), n \geq 0$ so that $P=B \tilde{P}$ and $(x-\lambda) \tilde{P}=A P$, which are just (2.19) and (2.20).

Let $\left\{P_{n}(x)\right\}_{n=0}^{\infty}$ and $\left\{\tilde{P}_{n}(x)\right\}_{n=0}^{\infty}$ be two MOPS's relative to $\sigma$ and $\tilde{\sigma}$ respectively. If $\tilde{\sigma}=(x-\lambda) \sigma$, then there are non-zero constants $\alpha_{n}, n \geq 0$ and $\beta_{n}, n \geq 1$ such that (2.19) and (2.20) hold (see Lemma 2.2).

Conversely we have:

Proposition 2.6. Let $\left\{P_{n}(x)\right\}_{n=0}^{\infty}$ and $\left\{\tilde{P}_{n}(x)\right\}_{n=0}^{\infty}$ be two monic $P S$ 's.

(i) If there are constants $\lambda$ and $\alpha_{n} \neq 0, \quad n \geq 0$ such that (2.20) holds, then $(x-\lambda) \sigma$ is a canonical moment functional of $\left\{\tilde{P}_{n}(x)\right\}_{n=0}^{\infty}$, where $\sigma$ is a canonical moment functional of $\left\{P_{n}(x)\right\}_{n=0}^{\infty}$.

(ii) If there are constants $\lambda$ and $\alpha_{n} \neq 0(n \geq 0), \beta_{n} \neq 0(n \geq 1)$ such that (2.19) and (2.20) hold, then both $\left\{P_{n}(x)\right\}_{n=0}^{\infty}$ and $\left\{\tilde{P}_{n}(x)\right\}_{n=0}^{\infty}$ are monic OPS's relative to $\sigma$ and $\tilde{\sigma}=(x-\lambda) \sigma$ respectively and

$$
P_{n}(\lambda) \neq 0, \quad n \geq 1 \text {. }
$$

Proof. (i) By (2.20), we have

$$
\left\langle(x-\lambda) \sigma, \tilde{P}_{n}(x)\right\rangle=\left\langle\sigma, P_{n+1}(x)+\alpha_{n} P_{n}(x)\right\rangle=\alpha_{0} \sigma_{0} \delta_{0 n}, \quad n \geq 0
$$

so that $(x-\lambda) \sigma$ is a canonical moment functional of $\left\{\tilde{P}_{n}(x)\right\}_{n=0}^{\infty}$. 
(ii) By (2.19) and (2.20), we have

$$
\begin{aligned}
x P_{n}(x)= & P_{n+1}(x)+\left(\alpha_{n}+\beta_{n}+\lambda\right) P_{n}(x) \\
& +\alpha_{n-1} \beta_{n} P_{n-1}(x), \quad n \geq 0\left(\beta_{0}=0\right) \\
x \tilde{P}_{n}(x)= & \tilde{P}_{n+1}(x)+\left(\alpha_{n}+\beta_{n+1}+\lambda\right) P_{n}(x) \\
& +\alpha_{n} \beta_{n} \tilde{P}_{n-1}(x), \quad n \geq 0
\end{aligned}
$$

so that $\left\{P_{n}(x)\right\}_{n=0}^{\infty}$ and $\left\{\tilde{P}_{n}(x)\right\}_{n=0}^{\infty}$ are MOPS's relative to $\sigma$ and $\tilde{\sigma}=$ $(x-\lambda) \sigma$ (by (i)) respectively by Farvard' theorem ([2]). Finally, $P_{n}(\lambda) \neq$ $0, n \geq 1$ by Lemma 2.2 .

Theorem 2.7. The Jacobi matrix $J=\left[c_{n}, b_{n}, 1\right]$ of the MOPS $\left\{P_{n}(x)\right\}_{n=0}^{\infty}$ can be decomposed into $J=B A+\lambda I$ if and only if the free parameter $\lambda$ satisfies

$$
P_{n}(\lambda) \neq 0, \quad n \geq 1 .
$$

Moreover, in this case, the corresponding Darboux transform $\left\{\tilde{P}_{n}(x)\right\}_{n=0}^{\infty}$ is an MOPS relative to $\tilde{\sigma}=(x-\lambda) \sigma$ and

$$
\alpha_{n}=-\frac{P_{n+1}(\lambda)}{P_{n}(\lambda)}, \quad n \geq 0 \quad \text { and } \quad \beta_{n}=-c_{n} \frac{P_{n-1}(\lambda)}{P_{n}(\lambda)}, \quad n \geq 1 .
$$

Proof. Assume that $J$ can be decomposed into $J=B A+\lambda I$. Then $c_{n}=\alpha_{n-1} \beta_{n}, n \geq 1$ by (2.17) so that $\alpha_{n} \neq 0, n \geq 0$ and $\beta_{n} \neq 0, n \geq$ 1. Hence by Lemma 2.5 and Proposition 2.6, $\left\{\tilde{P}_{n}(x)\right\}_{n=0}^{\infty}$ is an MOPS relative to $\tilde{\sigma}=(x-\lambda) \sigma$ and $P_{n}(\lambda) \neq 0, n \geq 1$.

Conversely assume $P_{n}(\lambda) \neq 0, n \geq 1$. Then by Lemma $2.2, \tilde{\sigma}=$ $(x-\lambda) \sigma$ is quasi-definite and (2.11) and (2.12) hold for the MOPS $\left\{\tilde{P}_{n}(x)\right\}_{n=0}^{\infty}$ relative to $\tilde{\sigma}$.

We can express (2.11) and (2.12) as

$$
(x-\lambda) \tilde{P}=A P \quad \text { and } \quad P=B \tilde{P},
$$

where $A=\left[0, \alpha_{n}, 1\right]$ and $B=\left[\beta_{n}, 1,0\right]$ with $\alpha_{n}$ and $\beta_{n}$ as in (2.21). Then $(J-\lambda) P=B A P$ so that $J=B A+\lambda I$.

In summary, we have two Darboux transforms:

- $J=A B+\lambda I \longrightarrow \tilde{J}=B A+\lambda I$, which transforms $\sigma$ into $\tilde{\sigma}$ with $(x-\lambda) \tilde{\sigma}=\sigma$, that is, $\tilde{\sigma}=(x-\lambda)^{-1} \sigma+\tilde{\sigma}_{0} \delta(x-\lambda)$ (cf. (1.1));

- $J=B A+\lambda I \longrightarrow \tilde{J}=A B+\lambda I$, which transforms $\sigma$ into $\tilde{\sigma}$ with $\tilde{\sigma}=(x-\lambda) \sigma$. 
In the literature [16], the transform from $\sigma$ into $\tilde{\sigma}=(x-\lambda)^{-1} \sigma+$ $\tilde{\sigma}_{0} \delta(x-\lambda)$ is called the Geronimus transform and the transform from $\sigma$ into $\tilde{\sigma}=(x-\lambda) \sigma$ is called the Christoffel transform.

Recall that in decomposition $J=A B+\lambda I$, we have two free parameter $\alpha_{0}$ and $\lambda$ but in decomposition $J=B A+\lambda I$, we have only one free parameter $\lambda$. Hence, if we perform these two Darboux transforms with fixed $\lambda$ in succession, then we have

- $J=A B+\lambda I \longrightarrow \tilde{J}=B A+\lambda I \quad \longrightarrow \quad \tilde{\tilde{J}}=A B+\lambda I$, which transforms $\sigma$ into $\sigma$ itself (that is, Christoffel transform is the left inverse of Geronimus transform).

- $J=B A+\lambda I \longrightarrow \tilde{J}=A B+\lambda I=A^{\prime} B^{\prime}+\lambda I \longrightarrow \tilde{\tilde{J}}=$ $B^{\prime} A^{\prime}+\lambda I$, which transforms $\sigma$ into $\tilde{\tilde{\sigma}}$ with $(x-\lambda) \tilde{\tilde{\sigma}}=(x-\lambda) \sigma$ so that $\tilde{\tilde{\sigma}}=\sigma+\left(\tilde{\tilde{\sigma}}_{0}-\sigma_{0}\right) \delta(x-\lambda)$.

As a point mass perturbation of $\sigma, \tilde{\tilde{\sigma}}=\sigma+\left(\tilde{\tilde{\sigma}}_{0}-\sigma_{0}\right) \delta(x-\lambda)$ is called a Uvarov transform of $\sigma([16])$.

This observation explains why the "Krall polynomials" (see [4] for definition) can be obtained from classical orthogonal polynomials via a succession of suitable Darboux transforms (see Theorem 2 in [4]) since Krall polynomials are orthogonal relative to a classical moment functional plus one or two point masses.

\section{Further results}

Let $\sigma$ be a quasi-definite moment functional. Then by a Darboux transform with a parameter $\lambda, \sigma$ is transformed into $\tilde{\sigma}$ satisfying either $(x-\lambda) \tilde{\sigma}=\sigma$ in case of Geronimus transform or $\tilde{\sigma}=(x-\lambda) \sigma$ in case of Christoffel transform. Then by a succession of Darboux transforms with varying parameters, $\sigma$ is transformed into $\tau$ satisfying

$$
r(x) \sigma=s(x) \tau
$$

where $r(x)$ and $s(x)$ are polynomials.

LEMMA 3.1. (cf. [9]) Let $\sigma$ and $\tau$ be quasi-definite moment functionals with the corresponding OPS's $\left\{P_{n}(x)\right\}_{n=0}^{\infty}$ and $\left\{Q_{n}(x)\right\}_{n=0}^{\infty}$, respectively. Then the followings are equivalent:

(i) $r(x) \sigma=s(x) \tau$ for some polynomials $r(x)$ of degree $r \geq 0$ and $s(x)$ of degree $s \geq 0$

(ii) there are an integer $s \geq 0$ and constants $a_{i}^{n}(n-s \leq i \leq n+r)$ and a polynomial $r(x)$ of degree $r \geq 0$ such that $a_{n-s}^{n} \neq 0$ and 


$$
r(x) Q_{n}(x)=\sum_{i=n-s}^{n+r} a_{i}^{n} P_{i}(x), \quad n \geq s
$$

(iii) there are an integer $r \geq 0$ and constants $b_{i}^{n}(n-r \leq i \leq n+s)$ and a polynomial $s(x)$ of degree $s \geq 0$ such that $b_{n-r}^{n} \neq 0$ and

$$
s(x) P_{n}(x)=\sum_{i=n-r}^{n+s} b_{i}^{n} Q_{i}(x), \quad n \geq r .
$$

Proof. Assume that (i) holds. Expand $r(x) Q_{n}(x)$ as

$$
r(x) Q_{n}(x)=\sum_{i=0}^{n+r} a_{i}^{n} P_{i}(x) .
$$

Then

$$
\begin{aligned}
& a_{k}^{n}\left\langle\sigma, P_{k}^{2}(x)\right\rangle \\
& =\left\langle\sigma, r(x) Q_{n}(x) P_{k}(x)\right\rangle \\
& =\left\{\begin{array}{cc}
\left\langle\tau, s(x) Q_{n}(x) P_{k}(x)\right\rangle=0, & k+s<n, \\
\text { non-zero, } & k+s=n .
\end{array}\right.
\end{aligned}
$$

Thus we have the relation (3.2).

Conversely assume that (3.2) holds. Then

$$
\begin{aligned}
& \left\langle r(x) \sigma, Q_{n}(x)\right\rangle \\
= & \left\langle\sigma, r(x) Q_{n}(x)\right\rangle \\
= & \left\{\begin{array}{cc}
\sum_{i=n-s}^{n+r} a_{i}^{n}\left\langle\sigma, P_{i}(x)\right\rangle=0, & n>s, \\
\text { non-zero, } & n=s .
\end{array}\right.
\end{aligned}
$$

Then Lemma 1.1 (iv) implies that there is a polynomial $s(x)$ of degree $s$ such that $r(x) \sigma=s(x) \tau$, which completes the equivalence of (i) and (ii). In the same fashion, we can show the equivalence of (i) and (iii), which proves the lemma.

REMARK. Let $F(\sigma)(x):=\sum_{n=0}^{\infty} \frac{\sigma_{n}}{x^{n+1}}$ be the (formal) Stieltjes function of $\sigma$. Then we can express (3.1) as

$$
F(\tau)(x)=\frac{r(x) F(\sigma)(x)+b(x)}{s(x)} \text { for some polynomial } b(x)
$$

so that $F(\tau)(x)$ is a linear spectral transform of $F(\sigma)(x)([16])$.

Note that equations (3.2) and (3.3) hold trivially for $0 \leq n \leq s-1$ and $0 \leq n \leq r-1$ with $P_{k}(x)=0$ for $k<0$. Hence we can express (3.2) 
and (3.3) as

$$
r(x) Q=A P \quad \text { and } \quad s(x) P=B Q
$$

where $A=\left(a_{k}^{n}\right)$ and $B=\left(b_{k}^{n}\right)$ are finite-band semi-infinite matrices and $P=\left(P_{0}(x), P_{1}(x), \cdots\right)^{t}, Q=\left(Q_{0}(x), Q_{1}(x), \cdots\right)^{t}$. Hence, we have:

Proposition 3.2. Let $\sigma$ and $\tau$ be quasi-definite moment functionals satisfying (3.1). Let $\left\{P_{n}(x)\right\}_{n=0}^{\infty}$ and $\left\{Q_{n}(x)\right\}_{n=0}^{\infty}$ be OPS's relative to $\sigma$ and $\tau$, respectively. Then there are finite-band semi-infinite matrices $A$ and $B$ satisfying

$$
\begin{aligned}
& r(x) s(x) P=B A P \quad \text { so that } B A=r\left(J_{P}\right) s\left(J_{P}\right), \\
& r(x) s(x) Q=A B Q \quad \text { so that } A B=r\left(J_{Q}\right) s\left(J_{Q}\right),
\end{aligned}
$$

where $J_{P}$ and $J_{Q}$ are the Jacobi matrices of $\left\{P_{n}(x)\right\}_{n=0}^{\infty}$ and $\left\{Q_{n}(x)\right\}_{n=0}^{\infty}$, respectively.

Proof. Let $A$ and $B$ be the same as in (3.4). Then

$$
r(x) s(x) P=r(x) B Q=B r(x) Q=B A P .
$$

Since $x P=J_{P} P, r(x) s(x) P=r\left(J_{P}\right) s\left(J_{P}\right) P=B A P$. Thus we have $r\left(J_{P}\right) s\left(J_{P}\right)=B A$, which proves (3.5). (3.6) is obtained in the similar fashion.

TheOrem 3.3. (cf. Theorem 3.1 and 3.2 in [13]) Let $\left\{P_{n}(x)\right\}_{n=0}^{\infty}$ be an OPS relative to $\sigma, r \geq 0$ an integer, and $L_{0}[\cdot]=\sum_{i=0}^{k} a_{i}(x) D^{i}(D=$ $d / d x)$ a $k$-th order linear differential operator with polynomial coefficients $a_{i}(x)$ of degree $\leq i$. If $\left\{Q_{n}(x)\right\}_{n=0}^{\infty}$ defined by

$$
Q_{n}(x):=L_{0}\left[P_{n+r}^{(r)}\right](x)=\sum_{i=0}^{k} a_{i}(x) P_{n+r}^{(i+r)}(x), \quad n \geq 0
$$

is also an OPS, then there are two finite-band semi-infinite matrices $A$ and $B$ and a linear differential operator $L_{2}[\cdot]$ with polynomial coefficients of order $k+r$ :

$$
L_{2}[\cdot]=\sum_{i=0}^{k+r} b_{i}(x) D^{i} \quad\left(\operatorname{deg}\left(b_{i}\right) \leq i+r\right)
$$

such that

$$
\left\{\begin{array}{l}
L_{2} \circ L_{1}\left[P_{n}\right](x)=\lambda_{n} P_{n}(x), \quad n \geq 0 \\
L_{1} \circ L_{2}\left[Q_{n}\right](x)=\lambda_{n+r} Q_{n}(x), \quad n \geq 0 \quad\left(\lambda_{n+r} \neq 0\right)
\end{array}\right.
$$


for some constants $\lambda_{0}=\lambda_{1}=\cdots=\lambda_{r-1}=0$ and $\lambda_{n} \neq 0, n \geq r$, where $L_{1}[\cdot]:=L_{0} \circ D^{r}$ and

$$
a_{k}(x) b_{k+r}(x) P=B A P, \quad a_{k}(x) b_{k+r}(x) Q=A B Q
$$

(so that $\left\{Q_{n}(x)\right\}_{n=0}^{\infty}$ is obtained from $\left\{P_{n}(x)\right\}_{n=0}^{\infty}$ by a succession of Darboux transforms).

Proof. Assume that $\left\{Q_{n}(x)\right\}_{n=0}^{\infty}$ is an OPS relative to $\tau$. We then claim that there are polynomials $\left\{b_{j}(x)\right\}_{j=0}^{k+r}$ with $\operatorname{deg}\left(b_{j}\right) \leq j+r$ and

$$
\sum_{i=j}^{k+r}(-1)^{i}\left(\begin{array}{l}
i \\
j
\end{array}\right)\left(a_{i-r}(x) \tau\right)^{(i-j)}=b_{j}(x) \sigma, \quad 0 \leq j \leq k+r,
$$

where $a_{i}(x)=0$ for $i<0$. First,

$$
\left\langle\tau, Q_{n}(x)\right\rangle=\left\langle\sum_{i=0}^{k+r}(-1)^{i}\left(a_{i-r}(x) \tau\right)^{(i)}, P_{n+r}(x)\right\rangle=0, \quad n \geq 1
$$

so that by Lemma 1.1 (ii), there is a polynomial $b_{0}(x)$ of degree $\leq r$, with which (3.9) holds for $j=0$. Assume now that there are polynomials $\left\{b_{j}(x)\right\}_{j=0}^{\ell}(0 \leq \ell<k+r)$ with $\operatorname{deg}\left(b_{j}\right) \leq j+r$ and (3.9) holds for $0 \leq j \leq \ell$. Then for $n \geq \ell+2$

$$
\begin{aligned}
0= & \left\langle\tau, Q_{\ell+1} Q_{n}\right\rangle \\
= & \left\langle\sum_{i=0}^{k+r}(-1)^{i}\left(Q_{\ell+1} a_{i-r} \tau\right)^{(i)}, P_{n+r}\right\rangle \\
= & \left\langle\sum_{j=0}^{k+r} Q_{\ell+1}^{(j)} \sum_{i=j}^{k+r}(-1)^{i}\left(\begin{array}{c}
i \\
j
\end{array}\right)\left(a_{i-r} \tau\right)^{(i-j)}, P_{n+r}\right\rangle \\
= & Q_{\ell+1}^{(\ell+1)}\left\langle\sum_{i=\ell+1}^{k+r}(-1)^{i}\left(\begin{array}{c}
i \\
\ell+1
\end{array}\right)\left(a_{i-r} \tau\right)^{(i-\ell-1)}, P_{n+r}\right\rangle \\
& +\left\langle\sigma,\left(\sum_{j=0}^{\ell} Q_{\ell+1}^{(j)} b_{j}\right) P_{n+r}\right\rangle \\
= & Q_{\ell+1}^{(\ell+1)}\left\langle\sum_{i=\ell+1}^{k+r}(-1)^{i}\left(\begin{array}{c}
i \\
\ell+1
\end{array}\right)\left(a_{i-r} \tau\right)^{(i-\ell-1)}, P_{n+r}\right\rangle
\end{aligned}
$$


so that

$$
\left\langle\sum_{i=\ell+1}^{k+r}(-1)^{i}\left(\begin{array}{c}
i \\
\ell+1
\end{array}\right)\left(a_{i-r} \tau\right)^{(i-\ell-1)}, P_{n+r}\right\rangle=0, \quad n \geq \ell+2 .
$$

Hence, again by Lemma 1.1 (iv), there is a polynomial $b_{\ell+1}(x)$ of degree $\leq \ell+r+1$ with which (3.9) holds for $j=\ell+1$, which proves the claim. From (3.9) for $j=k+r$, we have

$$
(-1)^{k+r} a_{k}(x) \tau=b_{k+r}(x) \sigma
$$

so that $b_{k+r}(x) \not \equiv 0$ and (3.8) follows from Proposition 3.2. On the other hand,

$$
\begin{aligned}
\left\langle\tau, Q_{n}^{2}\right\rangle & =\left\langle\sum_{j=0}^{k+r} Q_{n}^{(j)} \sum_{i=j}^{k+r}(-1)^{i}\left(\begin{array}{l}
i \\
j
\end{array}\right)\left(a_{i-r} \tau\right)^{(i-j)}, P_{n+r}\right\rangle \\
& =\left\langle\sigma,\left(\sum_{j=0}^{k+r} Q_{n}^{(j)} b_{j}\right) P_{n+r}\right\rangle \neq 0, \quad n \geq 0
\end{aligned}
$$

so that

$$
\sum_{j=0}^{k+r} b_{j, j+r} n_{(j)} \neq 0, \quad n \geq 0
$$

where $b_{j}(x)=\sum_{\ell=0}^{j+k} b_{j \ell} x^{\ell}$ and

$$
n_{(j)}=\left\{\begin{array}{cc}
1, & j=0 \\
n(n-1) \cdots(n-j+1), & j \geq 1
\end{array}\right.
$$

We now let

$$
\tilde{P}_{n}(x):=\left\{\begin{array}{cl}
P_{n}(x), & 0 \leq n \leq r-1 \\
L_{2}\left[Q_{n-r}\right](x), & n \geq r .
\end{array}\right.
$$

Then $\operatorname{deg}\left(\tilde{P}_{n}\right)=n, n \geq 0$ by (3.10) and we can show easily that $\left\{\tilde{P}_{n}(x)\right\}_{n=0}^{\infty}$ is an OPS relative to $\sigma$ by using

$$
\sum_{i=j}^{k+r}(-1)^{i}\left(\begin{array}{l}
i \\
j
\end{array}\right)\left(b_{i}(x) \sigma\right)^{(i-j)}=a_{j-r}(x) \tau, \quad 0 \leq j \leq k+r,
$$

which is a direct consequence of (3.9). Hence,

$$
\tilde{P}_{n}(x)=L_{2}\left[Q_{n-r}\right](x)=\lambda_{n} P_{n}(x), \quad n \geq r
$$


for some constants $\lambda_{n} \neq 0$. Finally,

$$
\begin{aligned}
L_{2} \circ L_{1}\left[P_{n}\right](x) & =L_{2} \circ L_{0}\left[P_{n}^{(r)}\right](x) \\
& =\left\{\begin{array}{cl}
0, & \text { if } 0 \leq n \leq r-1 \\
L_{2}\left[Q_{n-r}\right](x)=\lambda_{n} P_{n}(x), & \text { if } n \geq r
\end{array}\right.
\end{aligned}
$$

and

$$
L_{1} \circ L_{2}\left[Q_{n}\right](x)=\lambda_{n+r} L_{1}\left[P_{n+r}\right](x)=\lambda_{n+r} Q_{n}(x), \quad n \geq 0,
$$

which proves (3.7).

We now let $\left\{P_{n}(x)\right\}_{n=0}^{\infty}$ be an MOPS, which are eigenfunctions of a linear differential operator with polynomial coefficients:

$$
L\left[P_{n}(x)\right]=\sum_{i=0}^{N} \ell_{i}(x) P_{n}^{(i)}(x)=\lambda_{n} P_{n}(x), \quad n \geq 0
$$

where $\ell_{i}(x), 0 \leq i \leq N$, are polynomials of degree $\leq i$. Such an OPS is called a Bochner-Krall OPS $([11,12])$. We may express $(3.11)$ as

$$
L[P]=\Lambda P
$$

where $L[\cdot]=\sum_{i=0}^{N} \ell_{i}(x) D^{i}$ and $\Lambda=\operatorname{dial}\left(\lambda_{0}, \lambda_{1}, \cdots\right)$. Let

$$
\mathcal{B}=\langle J, \Lambda\rangle
$$

be the algebra of semi-infinite band matrices generated by the Jacobi matrix $J=\left[c_{n}, b_{n}, 1\right]$ of $\left\{P_{n}(x)\right\}_{n=0}^{\infty}$ and $\Lambda$, and

$$
\mathcal{B}^{\prime}=\langle x, L\rangle
$$

the algebra of differential operators with polynomial coefficients generated by $x$ and $L[\cdot]$.

Define an anti-isomorphism $b$ from $\mathcal{B}$ into $\mathcal{B}^{\prime}$ by

$$
b(J)=x, \quad b(\Lambda)=L, \quad \text { and } \quad b\left(J^{i} \Lambda^{j}\right)=L^{j} x^{i}
$$

for non-negative integers $i$ and $j$.

Theorem 3.4. (cf. Theorem 1 in [7]) Let $\left\{P_{n}(x)\right\}_{n=0}^{\infty}$ be a monic Bochner-Krall OPS satisfying (3.11) and $J$ the Jacobi matrix of $\left\{P_{n}(x)\right\}_{n=0}^{\infty}$, and let $\left\{Q_{n}(x)\right\}_{n=0}^{\infty}$ be an OPS relative to $\tau$. Then there is a nonzero polynomial $s(x)$ such that

(i) $s(J)=B C$, where $B$ and $C$ are finite-band semi-infinite matrices and $C \in \mathcal{B}$,

(ii) $C P=Q$ (so that $B C P=s(x) P$, and $C B Q=s(x) Q)$ 
if and only if there is a linear differential operator $L_{1}[\cdot] \in \mathcal{B}^{\prime}$ such that

$$
L_{1}\left[P_{n}(x)\right]=Q_{n}(x), \quad n \geq 0 .
$$

In this case, there is a linear differential operator $L_{2}[\cdot]$ of the same order as $L_{1}[\cdot]$ such that

$$
L_{2} \circ L_{1}\left[P_{n}(x)\right]=\mu_{n} P_{n}(x)
$$

and

$$
L_{1} \circ L_{2}\left[Q_{n}(x)\right]=\mu_{n} Q_{n}(x), \quad n \geq 0 \quad\left(\mu_{n} \neq 0, \quad n \geq 0\right) .
$$

(Hence $\left\{Q_{n}(x)\right\}_{n=0}^{\infty}$ is also a Bochner-Krall OPS, which is obtained from $\left\{P_{n}(x)\right\}_{n=0}^{\infty}$ by a succession of Darboux transforms).

Proof. $(\Rightarrow)$ Let $b(C):=L_{1}[\cdot] \in \mathcal{B}^{\prime}$. Then $L_{1}[P]=b(C) P=C P=$ $Q$, that is, $L_{1}\left[P_{n}(x)\right]=Q_{n}(x), n \geq 0$, which implies that $L_{1}[\cdot]=$ $\sum_{i=0}^{k} a_{i}(x) D^{i}$ is a linear differential operator with polynomial coefficients $a_{i}(x)$ of degree $\leq i$. Then by Theorem 3.3, there is a linear differential operator $L_{2}[\cdot]=\sum_{i=0}^{k} b_{i}(x) D^{i}$ of the same order as $L_{1}[\cdot]$ with polynomial coefficients $b_{i}(x)$ of degree $\leq i$ such that

$$
L_{2} \circ L_{1}\left[P_{n}(x)\right]=\mu_{n} P_{n}(x) \text { and } L_{1} \circ L_{2}\left[Q_{n}(x)\right]=\mu_{n} Q_{n}(x), \quad n \geq 0
$$

for some constants $\mu_{n} \neq 0, n \geq 0$.

$(\Leftrightarrow)$ Since $L_{1}[\cdot] \in \mathcal{B}^{\prime}$, there is a matrix $C \in \mathcal{B}$ such that $b(C)=L_{1}[\cdot]$. Then we have,

$$
Q=L_{1}[P]=C P
$$

By Theorem 3.3, there are two polynomials $r(x)$ and $s(x)$ such that $r(x) \sigma=s(x) \tau$. From Proposition 3.2, there are two finite band semiinfinite matrices $A$ and $B$ such that

$$
r(x) Q=A P \quad \text { and } \quad s(x) P=B Q .
$$

Hence we have by (3.12)

$$
s(J) P=s(x) P=B Q=B C P
$$

so that $s(J)=B C$ and $C \in \mathcal{B}$.

\section{References}

[1] S. Bochner, Über Sturm-Liouvillesche Polynomsysteme, Math. Z. 29 (1929), 730736.

[2] T. S. Chihara, An Introduction to Orthogonal Polynomials, Gordon and Breach, New York, 1977.

[3] _ , On co-recursive orthogonal polynomials, Proc. Amer. Math. Soc. 8 (1962), 899-905. 
[4] F. A. Grünbaum and L. Haine, Orthogonal polynomials satisfying differential equations: the role of the Darboux transformation, in: D. Levi, L. Vinet, P. Winternitz (Eds.), Symmetries and Integrability of Differential Equations, Estérel, 1994, CRM Proc. Lecture Notes, vol. 9, Amer. Math. Soc., Providence, RI, 1996, 143-154.

[5] — A theorem of Bochner, revisited, in: A. S. Fokas, I. M. Gelfand (Eds.), Algebraic Aspects of Integrable Systems: In memory of Irene Dorfman, Progr. Nonlinear Differential Equations, vol. 26, Birkhäuser, Boston, 1996, 143-172.

[6] _ Bispectral Darboux transformation: An extention of the Krall polynomials, IMRN (Internat. Mat. Res. Notices) 8 (1997), 359-392.

[7] F. A. Grünbaum, L. Haine, and E. Horozov, Some functions that generalize the Krall-Laguerre polynomials, J. Comput. Appl. Math. 106 (1999), 271-297.

[8] H. L. Krall, On orthogonal polynomials satisfying a certain fourth order differential equation, The Pennsylvania State College Studies No.6, The Penn. State college, Pa., 1940.

[9] D. H. Kim, K. H. Kwon, D. W. Lee, and F. Marcellán, Compatible pairs of orthogonal polynomials, Bull. Korean Math. Soc. 36 (1999), 779-797.

[10] K. H. Kwon, D. W. Lee, F. Marcellán and S. B. Park, On kernel polynomials and self perturbation of orthogonal polynomials, Annali di Matem. Pura ed Appl., to appear.

[11] K. H. Kwon, L. L. Littlejohn, and B. H. Yoo, Characterizations of orthogonal polynomials satisfying differential equations, SIAM J. Math. Anal. 25 (1994), no. 3, 976-990.

[12] K. H. Kwon, L. L. Littlejohn, and G. J. Yoon, Orthogonal Polynomial Solutions of Spectral Type Differential Equations: Magnus' Conjecture, J. Approximation Theory 112 (2001), no. 2, 189-215.

[13] K. H. Kwon and G. J. Yoon, Generalized Hahn's theorem, J. Comput. Appl. Math. 116 (2000), 243-262.

[14] L. L. Littlejohn, The Krall polynomials:a new class of orthogonal polynomials, Quaestiones Math. 5 (1982), 255-265.

[15] F. Marcellán and J. Petronilho, Orthogonal polynomials and coherent pairs : the classical case, Indag. Math., N. S. 6 (1995), 287-307.

[16] A. Zhedanov, Rational spectral transformations and orthogonal polynomials, J. Comput. Appl. Math. 85 (1997), 67-86.

Division of Applied Mathematics, KAIST, Taejon 305-701, Korea

E-mail: ykj@jacobi.kaist.ac.kr 